\title{
Application Site
}

National Cancer Institute

\section{Source}

National Cancer Institute. Application Site. NCI Thesaurus. Code C77676.

The anatomic site at which medical intervention is administered. 